\title{
ON SUBSPACES OF NON-REFLEXIVE ORLICZ SPACES
}

\author{
J. ALEXOPOULOS
}

May 28, 1997

ABSTRACT. Kadec and Pelczýnski have shown that every non-reflexive subspace of $L^{1}(\mu)$ contains a copy of $l_{1}$ complemented in $L^{1}(\mu)$. On the other hand Rosenthal investigated the structure of reflexive subspaces of $L^{1}(\mu)$ and proved that such subspaces, have non-trivial type. We show the same facts to hold true, for a special class of non-reflexive Orlicz spaces. In particular we show that if $F$ is an $N$-function in $\Delta_{2}$ with its complement $G$ satisfying $\lim _{t \rightarrow \infty} \frac{G(c t)}{G(t)}=\infty$ then every non-reflexive subspace of $L_{F}^{*}$, contains a copy of $l_{1}$ complemented in $L_{F}^{*}$. Furthermore we establish the fact that if $F$ is an $N$-function in $\Delta_{2}$ with its complement $G$ satisfying $\lim _{t \rightarrow \infty} \frac{G(c t)}{G(t)}=\infty$ then every reflexive subspace of $L_{F}^{*}$ has non trivial type.

Mathematics Subject Classification. 46E30. 


\section{Introduction and Background}

Kadec and Pelczýnski in [4] have shown that every non-reflexive subspace of $L^{1}(\mu)$ contains a copy of $l_{1}$ complemented in $L^{1}(\mu)$. On the other hand Rosenthal in [12] investigated the structure of reflexive subspaces of $L^{1}(\mu)$ and proved that such subspaces, have non-trivial type.

In this paper we will establish similar results for a special class of non-reflexive Orlicz spaces. In particular, in Section 2 we show that if $F$ is an $N$-function in $\Delta_{2}$ with its complement $G$ satisfying $\lim _{t \rightarrow \infty} \frac{G(c t)}{G(t)}=$ $\infty$ then every non-reflexive subspace of $L_{F}^{*}$, contains a copy of $l_{1}$ complemented in $L_{F}^{*}$ (Theorem 2.4). Furthermore we show that if $F$ is an $N$-function in $\Delta_{2}$ with its complement $G$ satisfying $\lim _{t \rightarrow \infty} \frac{G(c t)}{G(t)}=\infty$ then every reflexive subspace of $L_{F}^{*}$ has non trivial type (Theorem 2.7).

\subsection{N-Functions and Orlicz Spaces}

We begin with recalling some basic facts about N-functions and Orlicz Spaces. For a detailed account of these facts, the reader could consult chapters one and two in [5]. Throughout this paper $\mu$ denotes a probability.

Definition 1.1 Let $p:[0, \infty) \rightarrow[0, \infty)$ be a right continuous, monotone increasing function with

1. $p(0)=0$;

2. $\lim _{t \rightarrow \infty} p(t)=\infty$;

3. $p(t)>0$ whenever $t>0$;

then the function defined by

$$
F(x)=\int_{0}^{|x|} p(t) d t
$$

is called an $N$-function.

The following proposition gives an alternative view of $\mathrm{N}$-functions.

Proposition 1.1 The function $F$ is an $N$-function if and only if $F$ is continuous, even and convex with

1. $\lim _{x \rightarrow 0} \frac{F(x)}{x}=0$;

2. $\lim _{x \rightarrow \infty} \frac{F(x)}{x}=\infty$;

3. $F(x)>0$ if $x>0$. 
Definition 1.2 For an $N$-function $F$ define

$$
G(x)=\sup \{t|x|-F(t): t \geq 0\}
$$

Then $G$ is an $N$-function and it is called the complement of $F$.

Observe that $F$ is the complement of its complement $G$.

Definition 1.3 An $N$-function $F$ is said to satisfy the $\Delta_{2}$ condition $\left(F \in \Delta_{2}\right)$ if $\limsup _{x \rightarrow \infty} \frac{F(2 x)}{F(x)}<\infty$. That is, there is a $K>0$ so that $F(2 x) \leq K F(x)$ for large values of $x$.

Given an N-function $F$, the corresponding space of F-integrable functions is defined as follows.

Definition 1.4 For an $N$-function $F$ and a measurable $f$ define

$$
\mathbf{F}(f)=\int F(f) d \mu
$$

Let $L_{F}=\{f$ measurable $: \mathbf{F}(f)<\infty\}$. If $G$ denotes the complement of $F$ let

$$
L_{F}^{*}=\left\{f \text { measurable }:\left|\int f g d \mu\right|<\infty \quad \forall g \in L_{G}\right\}
$$

The collection $L_{F}^{*}$ is then a linear space. For $f \in L_{F}^{*}$ define

$$
\|f\|_{F}=\sup \left\{\left|\int f g d \mu\right|: \mathbf{G}(g) \leq 1\right\}
$$

Then $\left(L_{F}^{*},\|\cdot\|_{F}\right)$ is a Banach space, called an Orlicz space. Moreover, letting $\|\cdot\|_{(F)}$ be the Minkowski functional associated with the convex set $\left\{f \in L_{F}^{*}: \mathbf{F}(f) \leq 1\right\}$, we have that $\|\cdot\|_{(F)}$ is an equivalent norm on $L_{F}^{*}$, called the Luxemburg norm. Indeed, $\|f\|_{(F)} \leq\|f\|_{F} \leq 2\|f\|_{(F)}$, for all $f \in L_{F}^{*}$.

The following theorem establishes the fact that an Orlicz space is a dual space.

Theorem 1.2 Let $F$ be an $N$-function and let $E_{F}$ be the closure of the bounded functions in $L_{F}^{*}$. Then the conjugate space of $\left(E_{F},\|\cdot\|_{(F)}\right)$ is $\left(L_{G}^{*},\|\cdot\|_{G}\right)$, where $G$ is the complement of $F$.

Theorem 1.3 Let $F$ be an $N$-function and $G$ be its complement. Then the following statements are equivalent:

$$
\text { 1. } L_{F}^{*}=E_{F} \text {. }
$$


2. $L_{F}^{*}=L_{F}$.

3. The dual of $\left(E_{F},\|\cdot\|_{(F)}\right.$ is $\left(L_{G}^{*},\|\cdot\|_{G}\right)$.

4. $F \in \Delta_{2}$

Theorem 1.4 (Hölder's Inequality) For $f \in L_{F}^{*}$ and $g \in L_{G}^{*}$ we have

$$
\int|f g| d \mu \leq\|f\|_{F} \cdot\|g\|_{(G)} .
$$

Theorem 1.5 If $f \in L_{F}^{*}$ then

$$
\|f\|_{F}=\inf \left\{\frac{1}{k}(1+\mathbf{F}(k f)): k>0\right\} .
$$

It follows then that $f \in L_{F}^{*}$ if and only if there is $c>0$ so that $\mathbf{F}(c f)<\infty$.

Proposition 1.6 If $\|f\|_{F} \leq 1$ then $f \in L_{F}$ and $\mathbf{F}(f) \leq\|f\|_{F}$.

Recall that a subset $\mathcal{K}$ of $L^{1}(\mu)$ is called uniformly integrable if given $\varepsilon>0$ there is a $\delta>0$ so that $\sup \left\{\int_{E}|f| d \mu: f \in \mathcal{K}\right\}<\varepsilon$ whenever $\mu(E)<\delta$. Alternatively $\mathcal{K}$ is bounded and uniformly integrable if and only if given $\varepsilon>0$ there is an $N>0$ so that

$$
\sup \left\{\int_{[|f|>c]}|f| d \mu: f \in \mathcal{K}\right\}<\varepsilon \text { whenever } c \geq N .
$$

The classical theorem of Dunford and Pettis [2, page 93], identifies the bounded, uniformly integrable subsets of $L^{1}(\mu)$ with the relatively weakly compact sets. A concept similar to Uniform Integrability is that of equiabsolute continuity.

Definition 1.5 We say that a collection $\mathcal{K} \subset L_{F}^{*}$ has equi-absolutely continuous norms if

$$
\forall \varepsilon>0 \exists \delta>0 \text { so that } \sup \left\{\left\|\chi_{E} f\right\|_{F}: f \in \mathcal{K}\right\}<\varepsilon \text { whenever } \mu(E)<\delta \text {. }
$$

For $f \in L_{F}^{*}$ we say that $f$ has absolutely continuous norm if $\{f\}$ has equi-absolutely continuous norms.

The following result deal with the equi-absolute continuity of the norms.

Theorem 1.7 A function $f \in L_{F}^{*}$ has absolutely continuous norm if and only if $f \in E_{F}$.

The next two results resemble the theorem of Dunford and Pettis. For their proofs the reader should consult [1], Lemma 2.1 and Corollary 2.9 . 
Theorem 1.8 If $F \in \Delta_{2}$ and $\mathcal{K} \subset L_{F}^{*}$ then the following statements are equivalent:

I) The set $\mathcal{K}$ has equi-absolutely continuous norms.

II)The collection $\{F(f): f \in \mathcal{K}\}$ is uniformly integrable in $L^{1}$.

Theorem 1.9 Let $F \in \Delta_{2}$ and suppose that its complement $G$ satisfies

$$
\lim _{t \rightarrow \infty} \frac{G(c t)}{G(t)}=\infty \text { for some } c>0 .
$$

Then a bounded set $\mathcal{K} \subset L_{F}^{*}$ is relatively weakly compact if and only if $\mathcal{K}$ has equi-absolutely continuous norms.

\subsection{Banach Spaces with Type}

Denote by $\left(r_{n}\right)$, the sequence of Rademacher functions. Recall that for a positive integer $n, r_{n}:[0,1] \rightarrow$ $\{-1,1\}$ is defined by

- $r_{n}(1)=-1$.

- $r_{n}(t)=(-1)^{(i-1)}$ for $t \in\left[\frac{i-1}{2^{n}}, \frac{i}{2^{n}}\right)$, where $i=1, \ldots, 2^{n}$.

Definition 1.6 A Banach space $X$ is said to have type $p$, for some $1<p \leq 2$, if there is a constant $K$ so that

$$
\left(\int_{0}^{1}\left\|\sum_{i=1}^{n} r_{i}(t) x_{i}\right\|^{p} d t\right)^{\frac{1}{p}} \leq K\left(\sum_{i=1}^{n}\left\|x_{i}\right\|^{p}\right)^{\frac{1}{p}}
$$

for any $x_{1}, \ldots, x_{n} \in X$.

It turns out that type's presence in a Banach space, is ultimately connected with the space's finite dimensional structure. To be more specific, we need the following notion.

Definition 1.7 Let $\lambda \geq 1$ and $X$ be a Banach space. We say that $X$ contains $l_{1}^{n}$ 's $\lambda$-uniformly if for each positive integer $n$ there is an isomorphism $T: l_{1}^{n} \rightarrow X$ so that $\|T\| \cdot\left\|T^{-1}\right\| \leq \lambda$.

It is easy to see from the definition above that $X$ contains $l_{1}^{n}$ 's $\lambda$-uniformly if and only if for each positive integer $n, \exists x_{1}, \ldots, x_{n} \in B_{X}$ such that

$$
\left\|\sum_{i=1}^{n} a_{i} x_{i}\right\| \geq \frac{1}{\lambda} \sum_{i=1}^{n}\left|a_{i}\right|
$$

for all choices of scalars $a_{1}, \ldots, a_{n}$. 
Theorem 1.10 (Pisier) The following are equivalent for a Banach space $X$ :

1. For each $\lambda>1, X$ does not contain $l_{1}^{n}$ 's $\lambda$-uniformly.

2. For some $\lambda>1, X$ does not contain $l_{1}^{n}$ 's $\lambda$-uniformly.

3. The space $X$ has type $p$ for some $1<p \leq 2$.

For a proof of this theorem as well as a more detailed account and bibliography, the reader should consult [11] and [10, pages 31-40].

\section{The Main Results}

\subsection{Subspaces containing complemented $l_{1}$}

In this section we derive a theorem similar to the one of Kadec and Pelczýnski, about $L^{1}$ in [4] (see also [2, pages 94-98]).

Lemma 2.1 Let $\left(f_{n}\right)$ be a normalized disjointly supported sequence in $L_{F}^{*}$, where $F \in \Delta_{2}$ and its complement $G$ satisfies $\lim _{x \rightarrow \infty} \frac{G(c x)}{G(x)}=\infty$, for some $c>0$. Then there is a subsequence $\left(f_{n_{k}}\right)$ of $\left(f_{n}\right)$ so that

i. $\left(f_{n_{k}}\right)$ is equivalent to $l_{1}$ 's unit vector basis.

ii. The closed linear span of $\left(f_{n_{k}}\right)$ is complemented in $L_{F}^{*}$ by means of a projection of norm less than or equal to $4 c$.

iii. The coefficient functionals $\left(\phi_{k}\right)$ extend to all of the dual of $L_{F}^{*}$ and $\left\|\phi_{k}\right\| \leq 4$ for all positive integers $k$.

Proof : Let $E_{n}$ denote the support of $f_{n}$. For each positive integer $n$ choose $g_{n} \in L_{G}$ with $\int G\left(g_{n}\right) d \mu \leq 1$ so that $\int g_{n} f_{n} d \mu \geq \frac{1}{2}$. There is no harm in assuming that each $g_{n}$ is also supported on $E_{n}$.

Claim that $\int G\left(g_{n} / c\right) d \mu \rightarrow 0$ as $n \rightarrow \infty$. Fix $\varepsilon>0$. Since $\lim _{x \rightarrow \infty} \frac{G(c x)}{G(x)}=\infty$ then $\lim _{x \rightarrow \infty} \frac{G(x / c)}{G(x)}=0$. So we can choose $x_{0}>0$ so that $\frac{G(x / c)}{G(x)}<\frac{\varepsilon}{2}$ whenever $x \geq x_{0}$. Since the $E_{n}$ 's are pairwise disjoint and $\mu$ is a probability, we have that $\mu\left(E_{n}\right) \rightarrow 0$ as $n \rightarrow \infty$. So there is a positive integer $N$ so that $\mu\left(E_{n}\right)<\frac{\varepsilon}{2 G\left(x_{0} / c\right)}$ whenever $n \geq N$. So for $n \geq N$ we have

$$
\int G\left(g_{n} / c\right) d \mu=\int_{\left[\left|g_{n}\right|<x_{0}\right]} G\left(g_{n} / c\right) d \mu+\int_{\left[\left|g_{n}\right| \geq x_{0}\right]} G\left(g_{n} / c\right) d \mu
$$




$$
\begin{aligned}
& \leq G\left(x_{0} / c\right) \mu\left(E_{n}\right)+\frac{\varepsilon}{2} \int G\left(g_{n}\right) d \mu \\
& <\frac{\varepsilon}{2}+\frac{\varepsilon}{2}=\varepsilon
\end{aligned}
$$

and so the claim is established.

Now choose a subsequence $\left(n_{k}\right)$ of the positive integers so that $\sum_{k=1}^{\infty} \int G\left(\frac{g_{n_{k}}}{c}\right) d \mu \leq 1$. For any sequence of signs $\sigma=\left(\varepsilon_{k}\right)$ define $g_{\sigma}=\sum_{k=1}^{\infty} \varepsilon_{k} g_{n_{k}}$. Since the $g_{n_{k}}$ 's are disjointly supported, $g_{\sigma}$ is well defined. Furthermore

$$
\begin{aligned}
\int G\left(\frac{g_{\sigma}}{c}\right) d \mu & =\sum_{k=1}^{\infty} \int_{E_{n_{k}}} G\left(\frac{g_{\sigma}}{c}\right) d \mu \\
& =\sum_{k=1}^{\infty} \int_{E_{n_{k}}} G\left(\frac{\varepsilon_{k} g_{n_{k}}}{c}\right) d \mu \\
& =\sum_{k=1}^{\infty} \int_{E_{n_{k}}} G\left(\frac{g_{n_{k}}}{c}\right) d \mu \\
& \leq 1
\end{aligned}
$$

So $g_{\sigma} \in L_{G}^{*}$. Recall that the norm of $g_{\sigma}$ in $L_{G}^{*}$, is given by $\left\|g_{\sigma}\right\|_{G}=\inf \left\{\frac{1}{k}\left(1+\int G\left(k g_{\sigma}\right) d \mu\right): k>0\right\}$ and so it is easy to see that $\left\|g_{\sigma}\right\|_{G}$ remains constant as $\sigma$ varies. Denote this constant by $M$ and observe that

$$
M=\left\|g_{\sigma}\right\|_{G} \leq c\left(1+\int G\left(\frac{g_{\sigma}}{c}\right) d \mu\right)=c\left(1+\sum_{k=1}^{\infty} \int G\left(\frac{g_{n_{k}}}{c}\right) d \mu\right) \leq 2 c
$$

Now for $\left(a_{k}\right) \in l_{1}$ let $\sigma=\left(\operatorname{sign}\left(a_{k}\right)\right)$. Then

$$
\begin{aligned}
\left\|\sum_{k=1}^{\infty} a_{k} f_{n_{k}}\right\|_{F} & \geq \frac{1}{\left\|g_{\sigma}\right\|_{G}} \int\left(g_{\sigma} \sum_{k=1}^{\infty} a_{k} f_{n_{k}}\right) d \mu \\
& =\frac{1}{M} \int\left(\sum_{k=1}^{\infty}\left|a_{k}\right| g_{n_{k}} f_{n_{k}}\right) d \mu \\
& =\frac{1}{M} \sum_{k=1}^{\infty}\left|a_{k}\right| \int g_{n_{k}} f_{n_{k}} d \mu \\
& \geq \frac{1}{2 M} \sum_{k=1}^{\infty}\left|a_{k}\right|
\end{aligned}
$$

Hence (i) is established.

Now define for each $k$, a functional $\phi_{k}$ on all of $L_{F}^{*}$ by

$$
\phi_{k}(f)=\frac{1}{\int g_{n_{k}} f_{n_{k}} d \mu} \cdot \int g_{n_{k}} f d \mu
$$


and define $P: L_{F}^{*} \rightarrow L_{F}^{*}$ by

$$
P(f)=\sum_{k=1}^{\infty} \phi_{k}(f) f_{n_{k}}
$$

Then for $k=1,2, \ldots$

$$
\left\|\phi_{k}\right\| \leq 2\left\|g_{n_{k}}\right\|_{G} \leq 2 \cdot\left(1+\mathbf{G}\left(g_{n_{k}}\right)\right) \leq 4 .
$$

Furthermore $P$ is a projection of $L_{F}^{*}$ onto the closed linear span of $\left(f_{n_{k}}\right)$ with

$$
\begin{aligned}
\|P\| & =\sup _{\|f\|_{F} \leq 1}\left\|\sum_{k=1}^{\infty} \frac{\int g_{n_{k}} f d \mu}{\int g_{n_{k}} f_{n_{k}} d \mu} \cdot f_{n_{k}}\right\|_{F} \\
& \leq 2 \sup _{\|f\|_{F} \leq 1} \sum_{k=1}^{\infty} \int\left|g_{n_{k}} f\right| d \mu \\
& \leq 2 \sup _{\|f\|_{F} \leq 1}\left\|\left(\sum_{k=1}^{\infty}\left|g_{n_{k}}\right|\right)\right\|_{G} \cdot\|f\|_{F} \\
& =2 M \\
& \leq 4 c .
\end{aligned}
$$

And so our proof is complete.

We state now the following result in form of a lemma. Its proof can be found in [2, page 50].

Lemma 2.2 Let $\left(z_{n}\right)$ be a basic sequence in the Banach space $X$ with coefficient functionals $\left(z_{n}^{*}\right)$. Suppose that there is a bounded linear projection $P: X \rightarrow X$ onto the closed linear span $\left[z_{n}\right]$ of $\left(z_{n}\right)$. If $\left(y_{n}\right)$ is any sequence in $X$ for which

$$
\sum_{n=1}^{\infty}\|P\| \cdot\left\|z_{n}^{*}\right\| \cdot\left\|z_{n}-y_{n}\right\|<1
$$

then $\left(y_{n}\right)$ is a basic sequence equivalent to $\left(z_{n}\right)$ and the closed linear span $\left[y_{n}\right]$ of $\left(y_{n}\right)$ is also complemented in $X$.

Lemma 2.3 Let $\left(f_{n}\right)$ be a sequence in $L_{F}^{*}$ where $F \in \Delta_{2}$ and its complement $G$ satisfies $\lim _{x \rightarrow \infty} \frac{G(c x)}{G(x)}=\infty$ for some $c>0$. Suppose that for each $\varepsilon>0$ there is a positive integer $n_{\varepsilon}$ so that $\mu\left(\left[\left|f_{n_{\varepsilon}}\right| \geq \varepsilon\left\|f_{n_{\varepsilon}}\right\|_{F}\right]\right)<\varepsilon$. Then there is a subsequence $\left(r_{n}\right)$ of $\left(f_{n}\right)$ so that $\left(\frac{r_{n}}{\left\|r_{n}\right\|_{F}}\right)$ is equivalent to $l_{1}$ 's unit vector basis. Furthermore the closed linear span $\left[r_{n}\right]$ of $\left(r_{n}\right)$ is complemented in $L_{F}^{*}$.

Proof : First observe that if $f \in L_{F}^{*}, E=\left[|f| \geq \varepsilon\|f\|_{F}\right]$ and $K$ is the norm of the inclusion map $L_{G}^{*} \hookrightarrow L^{1}$ then

$$
\left\|\chi_{E} \frac{f}{\|f\|_{F}}\right\|_{F} \geq\left\|\frac{f}{\|f\|_{F}}\right\|_{F}-\left\|\chi_{E^{c}} \frac{f}{\|f\|_{F}}\right\|_{F}
$$




$$
\begin{aligned}
& =1-\frac{1}{\|f\|_{F}} \sup \left\{\left|\int g \chi_{E^{c}} f d \mu\right|: g \in L_{G} \text { and } \mathbf{G}(g) \leq 1\right\} \\
& \geq 1-\frac{1}{\|f\|_{F}} \sup \left\{\|g\|_{1} \cdot\left\|\chi_{E^{c}} f\right\|_{\infty}: g \in L_{G} \text { and } \mathbf{G}(g) \leq 1\right\} \\
& \geq 1-\frac{K}{\|f\|_{F}}\left\|\chi_{E^{c}} f\right\|_{\infty} \\
& \geq 1-\frac{K}{\|f\|_{F}}\|f\|_{F} \cdot \varepsilon \\
& =1-K \varepsilon .
\end{aligned}
$$

So using the hypothesis there is a measurable set $E_{1}$ and a positive integer $n_{1}$ so that

$$
\mu\left(E_{1}\right)<\frac{1}{16 c \cdot 4^{2} K} \text { and }\left\|\chi_{E_{1}} \frac{f_{n_{1}}}{\left\|f_{n_{1}}\right\|_{F}}\right\|_{F} \geq 1-\frac{1}{16 c \cdot 4^{2}} .
$$

Since $F \in \Delta_{2}$ then each $f \in L_{F}^{*}$ has an absolutely continuous norm. This fact together with the hypothesis again, yields a measurable $E_{2}$ and a positive integer $n_{2}>n_{1}$ so that

$$
\begin{gathered}
\mu\left(E_{2}\right)<\frac{1}{16 c \cdot 4^{3} K}, \\
\left\|\chi_{E_{2}} \frac{f_{n_{2}}}{\left\|f_{n_{2}}\right\|_{F}}\right\|_{F}>1-\frac{1}{16 c \cdot 4^{3}}
\end{gathered}
$$

and

$$
\left\|\chi_{E_{2}} \frac{f_{n_{1}}}{\left\|f_{n_{1}}\right\|_{F}}\right\|_{F}<\frac{1}{16 c \cdot 4^{3}} .
$$

Continue inductively to construct a subsequence $\left(g_{n}\right)$ of $\left(f_{n}\right)$ and a sequence of measurable sets $\left(E_{n}\right)$ so that

$$
\begin{gathered}
\mu\left(E_{n}\right)<\frac{1}{16 c \cdot 4^{n+1} K}, \\
\left\|\chi_{E_{n}} \frac{g_{n}}{\left\|g_{n}\right\|_{F}}\right\|_{F}>1-\frac{1}{16 c \cdot 4^{n+1}}
\end{gathered}
$$

and

$$
\sum_{k=1}^{n-1}\left\|\chi_{E_{n}} \frac{g_{k}}{\left\|g_{k}\right\|_{F}}\right\|_{F}<\frac{1}{16 c \cdot 4^{n+1}}
$$

Now let

$$
A_{n}=E_{n} \backslash \bigcup_{k=n+1}^{\infty} E_{k} \text { and } h_{n}=\frac{g_{n}}{\left\|g_{n}\right\|_{F}} \chi_{A_{n}}
$$

Then

$$
\begin{aligned}
\left\|\frac{g_{n}}{\left\|g_{n}\right\|_{F}}-h_{n}\right\|_{F} & =\left\|\chi_{A_{n}^{c}} \frac{g_{n}}{\left\|g_{n}\right\|_{F}}\right\|_{F} \\
& \leq\left\|\chi_{E_{n}^{c}} \frac{g_{n}}{\left\|g_{n}\right\|_{F}}\right\|_{F}+\left\|\chi_{E_{n} \backslash A_{n}} \frac{g_{n}}{\left\|g_{n}\right\|_{F}}\right\|_{F}
\end{aligned}
$$




$$
\begin{aligned}
& \leq \frac{1}{16 c \cdot 4^{n+1}}+\left\|\chi_{\bigcup_{k=n+1}^{\infty} E_{k}} \frac{g_{n}}{\left\|g_{n}\right\|_{F}}\right\|_{F} \\
& \leq \frac{1}{16 c \cdot 4^{n+1}}+\left\|\sum_{k=n+1}^{\infty} \chi_{E_{k}} \frac{g_{n}}{\left\|g_{n}\right\|_{F}}\right\|_{F} \\
& \leq \frac{1}{16 c \cdot 4^{n+1}}+\sum_{k=n+1}^{\infty}\left\|\chi_{E_{k}} \frac{g_{n}}{\left\|g_{n}\right\|_{F}}\right\|_{F} \\
& \leq \frac{1}{16 c \cdot 4^{n+1}}+\sum_{k=n+1}^{\infty} \frac{1}{16 c \cdot 4^{k+1}} \\
& <\frac{1}{16 c \cdot 4^{n}} .
\end{aligned}
$$

Thus

$$
\begin{aligned}
1 & \geq\left\|h_{n}\right\|_{F} \\
& =\left\|\chi_{A_{n}} \frac{g_{n}}{\left\|g_{n}\right\|_{F}}\right\|_{F} \\
& \geq\left\|\chi_{E_{n}} \frac{g_{n}}{\left\|g_{n}\right\|_{F}}\right\|_{F}-\left\|\chi_{\bigcup_{k=n+1}^{\infty} E_{k}} \frac{g_{n}}{\left\|g_{n}\right\|_{F}}\right\|_{F} \\
& \geq 1-\frac{1}{16 c \cdot 4^{n+1}}-\sum_{k=n+1}^{\infty}\left\|\chi_{E_{k}} \frac{g_{n}}{\left\|g_{n}\right\|_{F}}\right\|_{F} \\
& \geq 1-\frac{1}{16 c \cdot 4^{n+1}}-\sum_{k=n+1}^{\infty} \frac{1}{16 c \cdot 4^{k+1}} \\
& >1-\frac{1}{16 c \cdot 4^{n}} .
\end{aligned}
$$

And so

$$
\begin{aligned}
\left\|\frac{g_{n}}{\left\|g_{n}\right\|_{F}}-\frac{h_{n}}{\left\|h_{n}\right\|_{F}}\right\|_{F} & \leq\left\|\frac{g_{n}}{\left\|g_{n}\right\|_{F}}-h_{n}\right\|_{F}+\left\|h_{n}-\frac{h_{n}}{\left\|h_{n}\right\|_{F}}\right\|_{F} \\
& \leq \frac{1}{16 c \cdot 4^{n}}+\left(1-\left\|h_{n}\right\|_{F}\right) \\
& \leq \frac{1}{16 c \cdot 4^{n}}+\left(1-1+\frac{1}{16 c \cdot 4^{n}}\right) \\
& =\frac{2}{16 c \cdot 4^{n}} .
\end{aligned}
$$

By Lemma (2.1), there is a subsequence $\left(n_{k}\right)$ of the positive integers so that

- $\left(\frac{h_{n_{k}}}{\left\|h_{n_{k}}\right\|_{F}}\right)$ is equivalent to $l_{1}$ 's unit vector basis.

- The closed linear span $\left[h_{n_{k}}\right]$ of $\left(h_{n_{k}}\right)$ is complemented in $L_{F}^{*}$ by means of a projection $P$, of norm less than or equal to $4 c$.

- The coefficient functionals $\phi_{k}$ extend to all of $L_{G}^{*}$ with $\left\|\phi_{k}\right\|_{G} \leq 4$ for all $k$. 
So we have that if $r_{k}=g_{n_{k}}$ then

$$
\begin{aligned}
\sum_{k=1}^{\infty}\|P\| \cdot\left\|\phi_{k}\right\|_{G} \cdot\left\|\frac{r_{k}}{\left\|r_{k}\right\|_{F}}-\frac{h_{n_{k}}}{\left\|h_{n_{k}}\right\|_{F}}\right\|_{F} & \leq 16 c \cdot \sum_{k=1}^{\infty}\left\|\frac{g_{n_{k}}}{\left\|g_{n_{k}}\right\|_{F}}-\frac{h_{n_{k}}}{\left\|h_{n_{k}}\right\|_{F}}\right\|_{F} \\
& \leq 16 c \cdot \sum_{n=1}^{\infty}\left\|\frac{g_{n}}{\left\|g_{n}\right\|_{F}}-\frac{h_{n}}{\left\|h_{n}\right\|_{F}}\right\|_{F} \\
& \leq 16 c \cdot \sum_{n=1}^{\infty} \frac{2}{16 c \cdot 4^{n}} \\
& =\sum_{n=1}^{\infty} \frac{2}{4^{n}} \\
& <1 .
\end{aligned}
$$

Hence the result is established by an appeal to Lemma (2.2).

Theorem 2.4 Let $F \in \Delta_{2}$ with its complement $G$ satisfying

$$
\lim _{x \rightarrow \infty} \frac{G(c x)}{G(x)}=\infty \text { for some } c>0
$$

If $X$ is any non-reflexive subspace of $L_{F}^{*}$ then $X$ contains an isomorphic copy of $l_{1}$ that is complemented in $L_{F}^{*}$.

Proof: Since $X$ is not reflexive, then the ball $B_{X}$ of $X$ is not relatively weakly compact. Hence by Theorem (1.9), $B_{X}$ does not have equi-absolutely continuous norms. So by Theorem (1.8), the set $\left\{F(f): f \in B_{X}\right\}$ is not uniformly integrable in $L^{1}$. Thus there is a $\delta>0$ so that

$$
\lim _{a \rightarrow \infty} \sup \left\{\int_{[|f| \geq a]} F(f) d \mu ; f \in B_{X}\right\}=\delta .
$$

Keeping in mind that the above limit is actually an infimum we can find an increasing sequence $\left(a_{n}\right)$ of positive reals, with $a_{n} \rightarrow \infty$ as $n \rightarrow \infty$ so that

$$
\delta \leq \sup \left\{\int_{\left[|f| \geq a_{n}\right]} F(f) d \mu ; f \in B_{X}\right\}<\delta+\frac{1}{n},
$$

for each positive integer $n$. It follows then, that there is a sequence $\left(f_{n}\right)$ in $B_{X}$ so that

$$
\delta-\frac{1}{n}<\int_{\left[\left|f_{n}\right| \geq a_{n}\right]} F\left(f_{n}\right) d \mu<\delta+\frac{1}{n}
$$

for all positive integers $n$. Now let $g_{n}=f_{n} \chi_{\left[\left|f_{n}\right| \geq a_{n}\right]}$ and $h_{n}=f_{n}-g_{n}$. Observe that for each $\varepsilon>0$ we have

$$
\mu\left(\left[\left|g_{n}\right| \geq \varepsilon\left\|g_{n}\right\|_{F}\right]\right) \leq \mu\left(\left[\left|g_{n}\right|>0\right]\right)
$$




$$
\begin{aligned}
& \leq \mu\left(\left[\left|f_{n}\right| \geq a_{n}\right]\right) \\
& \leq \frac{1}{a_{n}} \int_{\left[\left|f_{n}\right| \geq a_{n}\right]}\left|f_{n}\right| d \mu \\
& \leq \frac{1}{a_{n}} \int_{\left[\left|f_{n}\right| \geq a_{n}\right]} F\left(f_{n}\right) d \mu \\
& \leq \frac{1}{a_{n}}
\end{aligned}
$$

provided that $n$ is large enough. Since $\frac{1}{a_{n}} \rightarrow 0$ as $n \rightarrow \infty$ then $\mu\left(\left[\left|g_{n}\right| \geq \varepsilon\left\|g_{n}\right\|_{F}\right]\right)<\varepsilon$ for even larger $n$. So by Lemma $(2.3),\left(g_{n}\right)$ has a subsequence that spans a complemented $l_{1}$ in $L_{F}^{*}$.

We now show that $\left(h_{n}\right)$ has equi-absolutely continuous norms. Note that if $m \leq n$ then $\left[\left|h_{m}\right| \geq a_{n}\right]=\emptyset$ while if $m>n$ then

$$
\begin{aligned}
\int_{\left[\left|h_{m}\right| \geq a_{n}\right]} F\left(h_{m}\right) d \mu & =\int_{\left[\left|f_{m}\right|<a_{m}\right] \cap\left[\left|f_{m}\right| \geq a_{n}\right]} F\left(f_{m}\right) d \mu \\
& =\int_{\left[\left|f_{m}\right| \geq a_{n}\right]} F\left(f_{m}\right) d \mu-\int_{\left[\left|f_{m}\right| \geq a_{m}\right]} F\left(f_{m}\right) d \mu \\
& \leq \sup \left\{\int_{\left[|f| \geq a_{n}\right]} F(f) d \mu: f \in B_{X}\right\}-\delta+\frac{1}{m} \\
& \leq \delta+\frac{1}{n}-\delta+\frac{1}{n} \\
& =\frac{2}{n}
\end{aligned}
$$

So for each positive integer $n$ we have

$$
\sup _{m} \int_{\left[\left|h_{m}\right| \geq a_{n}\right]} F\left(h_{m}\right) d \mu=\sup _{m>n} \int_{\left[\left|h_{m}\right| \geq a_{n}\right]} F\left(h_{m}\right) d \mu \leq \frac{2}{n} .
$$

It follows then that $\left\{F\left(h_{m}\right): m \geq 1\right\}$ is uniformly integrable in $L^{1}$ and so by Theorem $(1.8),\left(h_{n}\right)$ has equi-absolutely continuous norms as we claimed. Hence by Theorem $(1.9),\left(h_{n}\right)$ is relatively weakly compact in $L_{F}^{*}$. So by passing to appropriate subsequences, we can assume that $\left(g_{n}\right)$ spans a complemented $l_{1}$ in $L_{F}^{*}$ and $\left(h_{n}\right)$ is weakly convergent in $L_{F}^{*}$. Thus $\left(h_{2 n}-h_{2 n+1}\right)$ is weakly null. So by Mazur's theorem, there is an increasing sequence $\left(n_{k}\right)$ of positive integers and a sequence $\left(a_{k}\right)$ of non-negative reals so that

- $\sum_{j=n_{k}+1}^{n_{k+1}} a_{j}=1$.

- The sequence $\left(w_{k}\right)$ defined by $w_{k}=\sum_{j=n_{k}+1}^{n_{k+1}} a_{j}\left(h_{2 j}-h_{2 j+1}\right)$ is norm-null in $L_{F}^{*}$.

Let

$$
u_{k}=\sum_{j=n_{k}+1}^{n_{k+1}} a_{j}\left(f_{2 j}-f_{2 j+1}\right)
$$


and

$$
v_{k}=\sum_{j=n_{k}+1}^{n_{k+1}} a_{j}\left(g_{2 j}-g_{2 j+1}\right) .
$$

Then $u_{k}=v_{k}+w_{k}$ and $\left\|u_{k}-v_{k}\right\|_{F}=\left\|w_{k}\right\|_{F} \rightarrow 0$ as $k \rightarrow \infty$. By selection, $\left(\frac{g_{n}}{\left\|g_{n}\right\|_{F}}\right)$ was equivalent to $l_{1}$ 's unit vector basis with complemented span in $L_{F}^{*}$. As $\left\|g_{n}\right\|_{F} \geq \int F\left(g_{n}\right) d \mu \geq \delta-\frac{1}{n},\left(g_{n}\right)$ itself is equivalent to $l_{1}$ 's unit vector basis. A little thought convinces us that this is also the case with $\left(v_{k}\right)$, with the closed linear span of $\left(v_{k}\right)$ still complemented in $L_{F}^{*}$ of course. By passing to a subsequence to ensure that $\left\|u_{k}-v_{k}\right\|_{F}$ converges to zero fast enough to apply Lemma (2.2), the result is finished.

\subsection{Subspaces of $L_{F}^{*}$ that have type}

The work of Kadec and Pelczýnski in [4], finds its natural continuation in the work of Rosenthal. In [12], Rosenthal shows that a subspace of $L^{1}$ is reflexive if and only if it has non-trivial type. In this section, we follow his lead, to show that the same fact holds true for the special class of Orlicz spaces, we have been considering. The following result, mentioned in the form of a lemma, is due to Dor and Kauffman (see [3] ).

Lemma 2.5 Suppose $f_{1}, \ldots, f_{n} \in B_{L^{1}(\mu)}$ satisfy

$$
\left\|\sum_{i=1}^{n} a_{i} f_{i}\right\|_{1} \geq \theta \sum_{i=1}^{n}\left|a_{i}\right|
$$

for any $a_{1}, \ldots, a_{n}$, where $0<\theta<1$.

Then there exist pairwise disjoint measurable sets $A_{1}, \ldots, A_{n}$ such that

$$
\int_{A_{i}}\left|f_{i}\right| d \mu \geq \theta^{2}
$$

We now adapt that lemma to our purposes.

Lemma 2.6 Suppose $f_{1}, \ldots, f_{n} \in B_{L_{F}^{*}(\mu)}$ satisfy

$$
\left\|\sum_{i=1}^{n} a_{i} f_{i}\right\|_{F} \geq \theta \sum_{i=1}^{n}\left|a_{i}\right|
$$

for any $a_{1}, \ldots, a_{n}$, where $0<\theta<1$. Then there exist pairwise disjoint measurable sets $A_{1}, \ldots, A_{n}$ such that

$$
\left\|\chi_{A_{i}} f_{i}\right\|_{F} \geq \theta^{2} .
$$


Proof : There is no loss in assuming that $\left\|\sum_{i=1}^{n} a_{i} f_{i}\right\|_{F}>\theta \sum_{i=1}^{n}\left|a_{i}\right|$, provided that not all of $a_{1}, \ldots, a_{n}$ are zero. Choose now $g \in B_{L_{G}^{*}}$, where $G$ is the complement of $F$, so that

$$
\left|\int g\left(\sum_{i=1}^{n} a_{i} f_{i}\right) d \mu\right|>\theta \sum_{i=1}^{n}\left|a_{i}\right|
$$

Then

$$
\int\left|\sum_{i=1}^{n} a_{i}\left(g f_{i}\right)\right| d \mu>\theta \sum_{i=1}^{n}\left|a_{i}\right|
$$

and so by Lemma (2.5) there is a collection of measurable and pairwise disjoint sets $A_{1}, \ldots, A_{n}$ so that

$$
\int_{A_{i}}\left|g f_{i}\right| d \mu \geq \theta^{2} \quad \forall i=1, \ldots, n
$$

By Hölder's inequality we then have that for each $i=1, \ldots, n$

$$
\begin{aligned}
\left\|\chi_{A_{i}} f_{i}\right\|_{F} & \geq\|g\|_{G} \cdot\left\|\chi_{A_{i}} f_{i}\right\|_{F} \\
& \geq \int_{A_{i}}\left|g f_{i}\right| d \mu \\
& \geq \theta^{2}
\end{aligned}
$$

which is what we wanted.

The following theorem, characterizes reflexive subspaces of $L_{F}^{*}$, for $F \in \Delta_{2}$, with complement $G$ satisfying $\lim _{t \rightarrow \infty} \frac{G(m t)}{G(t)}=\infty$

Theorem 2.7 Let $F \in \Delta_{2}$, with its complement $G$ satisfying

$$
\lim _{t \rightarrow \infty} \frac{G(m t)}{G(t)}=\infty
$$

for some $m>0$. Let $X$ be a subspace of $L_{F}^{*}$. Then the following are equivalent :

1. The space $X$ is not reflexive.

2. The space $X$ contains a copy of $l_{1}$ complemented in $L_{F}^{*}$.

3. The space $X$ contains $l_{1}^{n}$ 's uniformly.

4. The space $X$ fails to have non-trivial type.

Proof : The implication " $1 \Rightarrow 2$ " is just theorem (2.4). As for " $2 \Rightarrow 3$ " it follows directly from the definitions. The double implication " $3 \Leftrightarrow 4$ " is Pisier's theorem (Theorem 1.10 ). So we will only show " $3 \Rightarrow 1$ ". 
Suppose that $X$ contains $l_{1}^{n}$ 's uniformly. Then there is a $0<\theta<1$ so that for each positive integer $n$, there are functions $f_{1}, \ldots, f_{n} \in B_{X}$ satisfying

$$
\left\|\sum_{i=1}^{n} a_{i} f_{i}\right\|_{F} \geq \theta \sum_{i=1}^{n}\left|a_{i}\right|
$$

for any choice of scalars $a_{1}, \ldots, a_{n}$. So by Lemma (2.6), we have that for each positive integer $n$, there are functions $f_{1}, \ldots, f_{n} \in B_{X}$ and measurable, pairwise disjoint sets $A_{1}, \ldots, A_{n}$ so that

$$
\left\|\chi_{A_{i}} f_{i}\right\|_{F} \geq \theta^{2} \quad i=1, \ldots, n .
$$

Since $A_{1}, \ldots, A_{n}$ are pairwise disjoint, at least one of them must have $\mu$-measure less than $\frac{1}{n}$. Thus $B_{X}$ cannot have equi-absolutely continuous norms. Hence by Theorem (1.9), $B_{X}$ is not weakly compact in $L_{F}^{*}$ and so $X$ is not reflexive.

\section{References}

[1] J. Alexopoulos, De La Vallée Poussin's Theorem and Weakly Compact Sets in Orlicz Spaces, QM 17 (1994), pp. 231-248.

[2] J. Diestel, Sequences and Series in Banach Spaces, Springer Verlag, 1984.

[3] L. E. Dor, On Embeddings of $L_{p}$-spaces in $L_{p}$-spaces, Ph.D dissertation, The Ohio State University, 1975.

[4] M. I. Kadec and A. Pelczýnski, Bases, Lacunary Sequences and Complemented Subspaces in the Spaces $L_{p}$, Studia Math. 21 (1962), pp. 161-176.

[5] M. A. Krasnoselskii and Ya. B. Rutickii, Convex functions and Orlicz Spaces, Noorhoff Ltd., Groningen, 1961.

[6] D. H. Leung, On the Weak Dunford-Pettis Property, Arch. Math 52 (1989), pp. 363-364.

[7] J. Lindenstrauss and L. Tzafriri, Classical Banach Spaces II. Function Spaces, A Series of Modern Surveys in Math. 97, Springer Verlag, 1979.

[8] W. A. J. Luxemburg, Banach Function Spaces, Delft 1955. 
[9] J. Musielak, Orlicz Spaces and Modular Spaces, Lecture Notes in Math. 1034, Springer Verlag, 1983.

[10] G. Pisier, Factorization of Linear Operators and Geometry of Banach Spaces, Conference Board of the Math. Sciences, Regional Conference Series in Math. 60, A.M.S., 1986.

[11] G. Pisier, Sur les espaces de Banach qui ne contiennent pas uniformément de $l_{n}^{1}$, C. R. Acad. Sci. Paris 277, Series A(1973), pp. 991-994.

[12] H. Rosenthal, On Subspaces of $L^{p}$, Annals of Math. 97 (1973), pp. 344-373.

KENT STATE UNIVERSITY, STARK CAMPUS, 6000 FRANK AV. NW, CANTON, OH 44720 